\title{
Resonant Exitation-A Possible Forcing of the Middle Atmosphere from Above and Below
}

\author{
A. A. KRIVOlutsKY \\ Central Aerological Observatory, Pervomayskaya str. 3, 141700 Dolgoprudny, Moscow Region, Russia
}

(Received November 9, 1994; Revised December 1, 1995; Accepted November 14, 1997)

\begin{abstract}
Tidal theory analysis, 2-D numerical modelling of a large-scale planetary transient waves excitation by external forcing and data analysis has revealed the possibility for resonant response of the middle atmosphere. Such reaction has a nature of a barotropic motions and looks like atmospheric Rossby waves. Two types of forcing has been realised in model runs: solar UV 27-day variability (from above) and tropospheric periodical disturbances (from below). The results of model calculations has shown that the middle atmosphere of the Earth has its own periods and may be excited by a weak signal. Some of these periods are near to solar rotation period and others to the meteorological periodicities (5-7 and 12-14 days). The amplitudes of the waves in terms of geopotential heights increase from below to the higher levels and reach the quantities of about $50-80 \mathrm{gpm}$ in the lower mesosphere. A special 2-D statistical method for transient waves detecting has shown that the real waves with similar spatial structure and character periods are really exist in the troposphere and lower stratosphere.
\end{abstract}

\section{Introduction}

The spectrum of the atmospheric oscillations study has a rather long history in relation to the atmospheric tides one (Haurwitz, 1940; Kato, 1966; Lindzen, 1968; Longuet-Higgins, 1968; Diky, 1969). Such mathematical procedure permits to separate a description of the vertical and horizontal wave's structure into two differential equations. The parameter of separation ( $h$ - "equivalent depth") is positive or negative for different cases and has a mathematical nature of the eigenvalue. Only single value for $h$ $=10 \mathrm{~km}$ (main resonance) one can use solving "vertical" equation to realize boundary conditions when the base temperature is constant. It was shown (Diky and Golitsyn, 1968) that results of theoretical values of Rossby waves velocities are near by to these calculated on base of real atmospheric oscillation analysis (Eliassen and Machenhauer, 1965) when $h=10 \mathrm{~km}$ is used. So that the possibility of resonant character of Rossby waves looks naturally (Ivanovsky and Krivolutsky, 1979; Shoeberl and Clark, 1980). When we take into account the middle atmosphere excitation such possibility needs the real sources. It is known that the troposphere is a region of strong planetary waves activity due to the baroclinic unstability and nonlinear processes and may to disturb the middle atmosphere at its lower boundary. The next possibility for disturbance is solar activity. For example the variations of atmospheric parameters with a period close to the period of solar rotation (27 days) in the troposphere of the Earth are well-known (Vitels, 1957; Smirnov, 1966; King et al., 1977). Then aerological data for lower stratosphere were took into account and revealed the similar periodicity (Ebel et al., 1981, 1986; Krivolutsky et al., 1987). Two-dimensional statistical waves-analysis has shown that these oscillations are caused by large-scale transient waves which has a wave number equals 1 and propagate practically without changing of phase with the height (Krivolutsky and Loshkova, 1989) like free modes. Theoretical provment of a solar nature for such effect was a very difficult task due to the absence of a real information about UV solar fluxes and good numerical models. The astronomical observations which gave the amplitude of about $0.1 \%$ for 27 -days period of solar constant has permitted to use such heating of the surface as a forcing inside tidal theory procedure (Volland, 1979). The results of calculations gave the amplitude of the wave an order of magnitude smaller 
than has obtained by King. Real information about UV (Heath, 1980) was used to estimate the amplitude of the 27-days tide which looks like Rossby wave gave the results closer to statistical analysis (Krivolutsky, 1989). The simultaneous observation of ozone mixing ratio distribution together with UV flux variations due to solar rotation were carried out (Hood, 1984; Gille et al., 1985; Keating et al., 1985) and revealed 27-days and 13.5-days periodicity in ozone accompanied by a similar oscillation in the 205 nm range of solar UV-radiation and temperature (Keating et al., 1987). Lidar measurements has shown the temperature changes on the time scale of the sun's rotation (Keckhut and Chanin, 1992). Photochemical modelling gave a good agreement for ozone oscillations $-0.5 \%$ for $1 \% \mathrm{UV}$ variability and has not such agreement for temperature oscillations (were twice more than in model calculations). It was supposed (Hood, 1987) that the dynamical effects may be important. Recently the 2-D numerical model for transient waves was used (Krivolutsky and Kiryushov, 1992) for calculation the reaction of the middle atmosphere to the 27-days periodic heating due to ozone absorption. The results revealed the resonant reaction of atmosphere near 25-30 days interval and gave a good agreement to statistical analysis. The given paper presents the results which support the idea of resonant forcing of the middle atmosphere.

\section{2-D Modelling}

Tidal theory does not permit to use the real spatial structure of zonal wind and a numerical model is needed. 2-D numerical model which based on quasigeostrophic vorticity equation, the energy conservation equation and the continuity equation. The equation for a stream function looked like as follow (Shoeberl and Clark, 1980; Krivolutsky and Kiryushov, 1992)

$$
\frac{\sin ^{2} \theta}{\cos \theta} \frac{\partial}{\partial \theta} \frac{\cos \theta}{\sin ^{2} \theta} \frac{\partial \psi}{\partial \theta}+\hat{L} \psi+\frac{\sin ^{2} \theta}{S^{2}} \frac{v_{2}}{v_{1}} \frac{\partial^{2} \psi}{\partial z^{2}}-\frac{i \sin ^{2} \theta}{S^{2} v_{1}} \frac{\partial \alpha}{\partial z} \frac{\partial \psi}{\partial z}=-\frac{i \sin ^{2} \theta}{v_{1}} k e^{z} \frac{\partial}{\partial z}\left(e^{-z} \frac{Q^{\prime}}{S^{2}}\right)
$$

where

$$
\begin{aligned}
& L=\frac{m}{v_{1}}\left[2 \Omega-\frac{1}{\cos ^{2} \theta} \frac{\partial}{\partial \theta}\left(\varpi \cos ^{2} \theta\right)-\sin ^{2} \theta e^{2} \times \frac{\partial}{\partial z}\left(\frac{e^{-z}}{S^{2}} \frac{\partial \varpi}{\partial z}\right)\right]-\frac{m^{2}}{\cos ^{2} \theta}-\frac{\sin ^{2} \theta}{S^{2}} \frac{v_{2}}{v_{1}} \frac{1}{2 S^{2}}, \\
& S^{2}=\frac{N^{2}}{(2 \Omega a)^{2}}, \quad v_{1}=\sigma+m \varpi-i r, \quad v_{2}=\sigma+m \varpi-i \alpha, \quad \sigma=\frac{\bar{U}}{a \cos \theta} .
\end{aligned}
$$

$N$-Brunt-Vaisala frequency; $\sigma$ - frequency; $r, \alpha$-Rayley friction and Newtonian cooling coefficients (Fig. 7); $a$ - radius of the Earth; $Q^{\prime}$ - external heat source; $\Omega$ - speed of Earth's rotation.

Equation was solved for $m=1,2,3$ and for different $\sigma$. There were two scenarios for model runs. For the first scenario the vertical velocity at the upper and down boundaries was equaled zero and the external forcing was realized inside the region of integration and was conditioned by solar 27-day UV variability and ozone non-zonal heating existance. For the second scenario the external periodic source was placed at the lower boundary and imitated a tropospheric influence. The numerical simulations were based on the fact that at the equator and at the pole the amplitude of the wave was equaled zero. The equation was solved from $500 \mathrm{mb}$ level up to $80 \mathrm{~km}$ and from equator to pole. A vertical grid distance was about $2.5 \mathrm{~km}$ and meridional grid distance was about $1.8^{\circ}$. To receive solution the numerical scheme of Madala (1978) was used.

It is a problem to formulate the source of exitation for model runs like a transient wave when we find the solution in similar form. It is necessary to solve this problem when we take into account the solar periodic influence. Fortunately nature gives the possibility to do it. In this case there are two important 


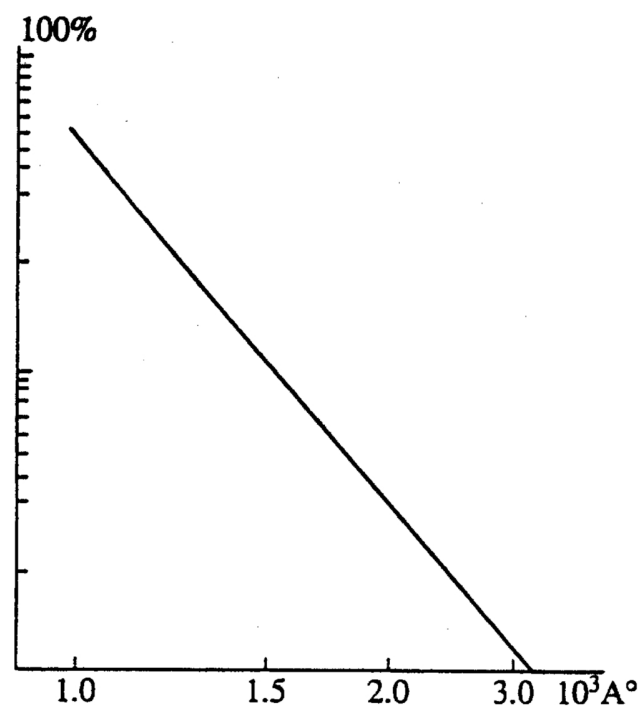

Fig. 1. 27-day oscillations in the solar UV flux for different wave's length of irradiance (Heath, 1980).

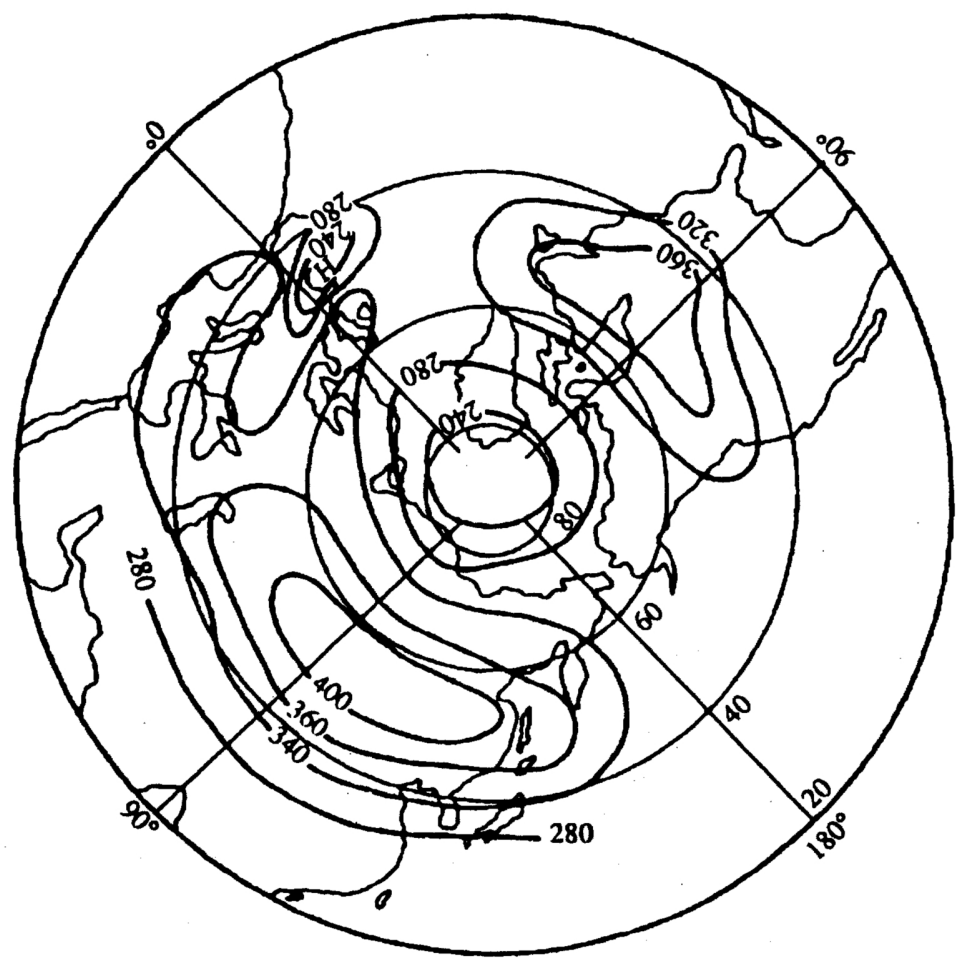

Fig. 2. Typical climatological picture of non-zonal ozone distribution (winter, Northern Hemisphere). 
points: solar UV periodical variability and ozone non-zonal behaviour. As we know the ozone heating function looks like as follow (Leovy, 1975)

$$
q(z, \lambda, \theta)=\frac{1}{T_{0}} \int_{0}^{t_{\text {day }}} \sum_{\lambda} \varepsilon_{\lambda} I_{\lambda_{\infty}} \sigma_{\lambda}\left[n_{\mathrm{O}_{3}}\right] e^{-\tau_{\mathrm{O}_{3}} / \cos Z} d t
$$

where $I_{\lambda_{\infty}}$ - solar UV radiation, $\sigma_{\lambda}$ - cross-sections of ozone absorption, $\varepsilon_{\lambda}$ - probability of the photolysis reaction, $\tau_{\mathrm{O}_{3}}$ - optical depth for ozone due to absorption, $Z$ - zenit angle, $t_{\text {day }}$ - sunlit duration, $T_{0}=24$ hours.

The rate of ozone heating in Eq. (2) is determined by the next way

$$
Q=\frac{q}{c_{p} \rho_{\mathrm{air}}}=\frac{\partial T}{\partial t},
$$

where $\rho_{\text {air }}$ - air density, $T$ - air temperature, $c_{p}$ - heat capacity.

As mentioned above the solar UV irradiance has 27-day periodicity and ozone content has non-zonal features (see Figs. 1 and 2). So that the linearized source of 27-day heating one can write like as follow

$$
Q^{\prime}=\frac{\Delta I_{\lambda_{\infty}}}{I_{\lambda_{\infty}}} \frac{\Delta n_{\mathrm{O}_{3}}}{n_{\mathrm{O}_{3}}} Q \cos (m \lambda) \cos (\sigma t) .
$$

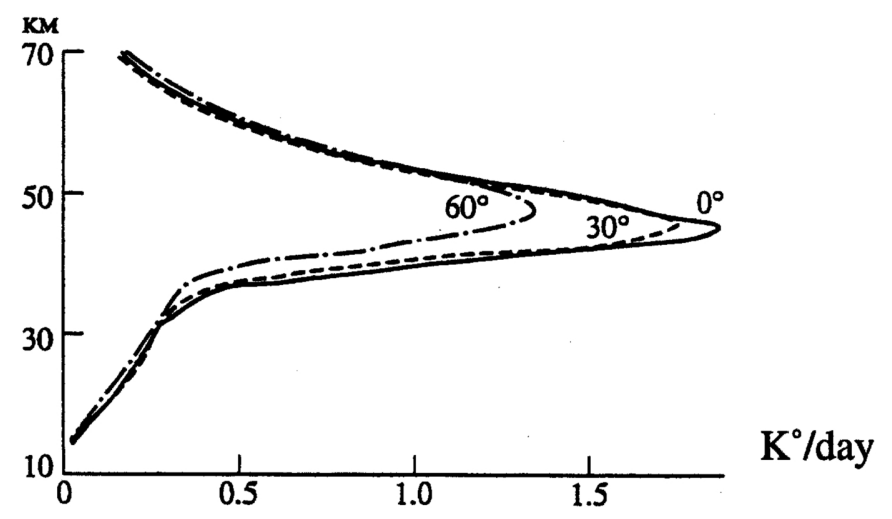

Fig. 3. Non-zonal part of ozone heating for different zenith angles of the sun due to the absorption in Huggins bands (assumed that nonzonal deviation of ozone relatively to zonalmean value equals $70 \%$ ).

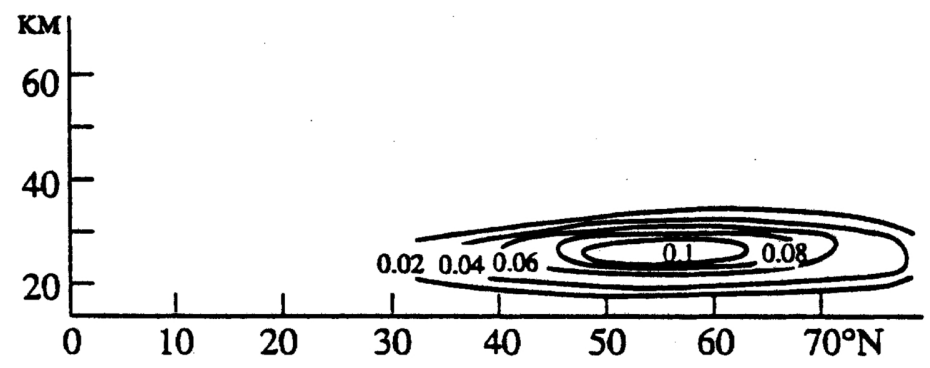

Fig. 4. Spatial structure of 27-day periodical heating was used in 2-D model calculations (deg/day). 
Such expression looks like a standing wave and may be rewrited formally in form of two transient waves superposition. These waves propagate into the opposite directions as follow

$$
Q^{\prime}=\frac{A}{2}\{\cos (m \lambda-\sigma t)+\cos (m \lambda+\sigma t)\}
$$

The analysis of the eigencurves behaviour for tidal theory equations shows that only one of these two waves may be practically exited when the "equivalent depth" is near by $10 \mathrm{~km}$ (main resonance). So that the source looking like standing wave generates transient planetary waves in the Earth's atmosphere when the resonant conditions are real. The useful parameterization of the ozone heating (Shoeberl and Strobel, 1978) was used to calculate a non-zonal part. Only Huggins bands absorption was took into account due to deepper penetration of UV solar radiation into the Earth's atmosphere for this area of solar spectrum. Figure 3 shows the nonzonal ozone heating for different solar zenith angles when the ratio of the ozone maximum to its minimum along fixed latitude equals 1.5 . One can see that a nonzonal heading in such situation would be about 1.5 degrees per day. So that a transient part of the source caused by 27 -day UV periodicity would be of about $0.1 \mathrm{deg} / \mathrm{day}$. Figure 4 shows the spatial structure of a source $\left(Q^{\prime}\right)$ which was used in model calculations. The maximum of this source was placed near by $25 \mathrm{~km}$ level (maximum of ozone concentration) and 50-60 deg. of latitudes (usual displacement of non-zonal disturbances of ozone in the Northern Hemisphere). The amplitude of the source at Fig. 4 was strongly reduced in model runs for the altitudes above $30 \mathrm{~km}$ due to the absence of information about non-zonal behaviour of ozone for higher altitudes.

\section{Results of Model Runs and Data Analysis}

Figures 5 and 6 show the results of 2-D model calculations in which the described above spatial structure of external solar source was used. It is necessary to mention that the parameters of dissipation (Fig. 7-Newtonian cooling and Rayley friction coefficients) were took in accordance to these which were used to 2-D numerical modelling of photochemistry and dynamics (Garsia and Solomon, 1983). Zonal wave numbers were equaled 1, 2, 3 in model runs. The period of exitation was changed in different model runs and so that the resonant curves for different layers of the middle atmosphere were calculated. Figures

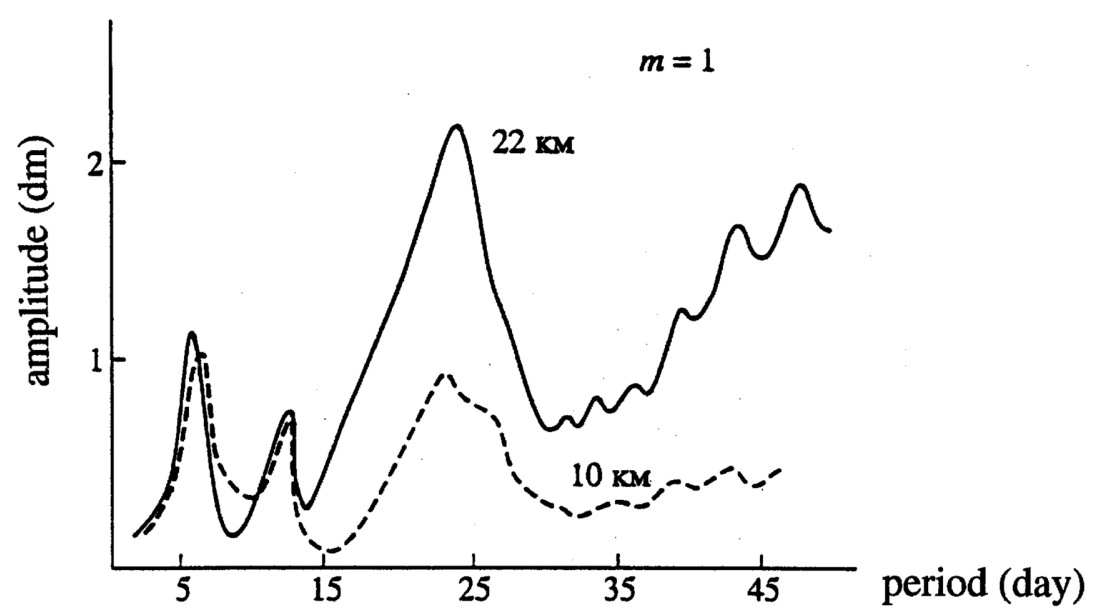

Fig. 5. Middle atmospheric response to solar signal at 10, $22 \mathrm{~km}$ and $60 \mathrm{~N}$ (spatial structure of the source is at Fig. 4), 2-D model runs. 


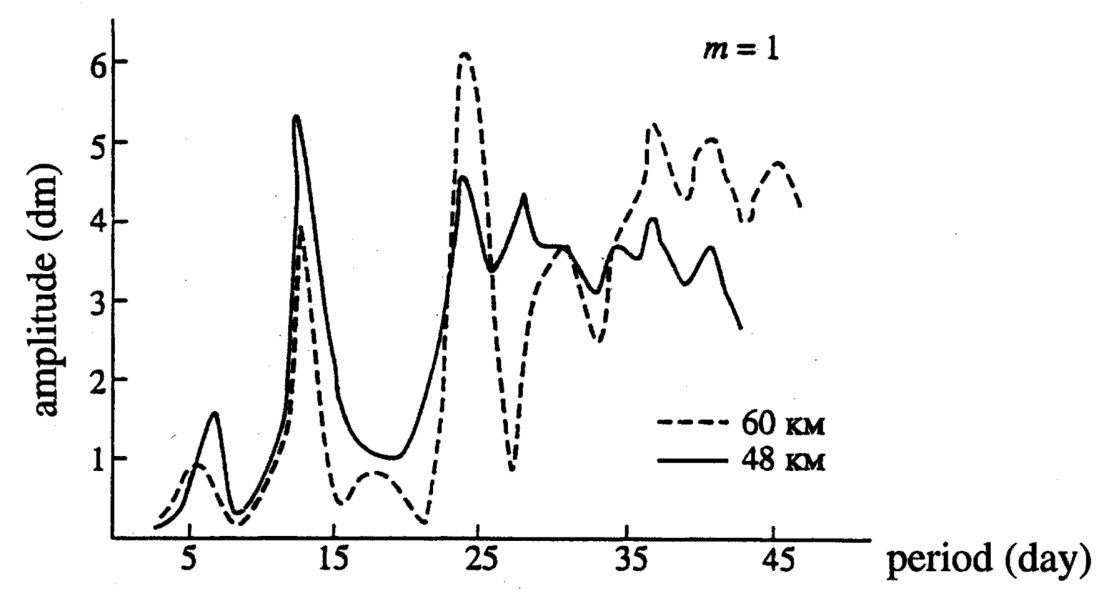

Fig. 6. Middle atmospheric response to solar signal at 48, $60 \mathrm{~km}$ and $60 \mathrm{~N}$ (spatial structure of the source is at Fig. 4), 2-D model runs.

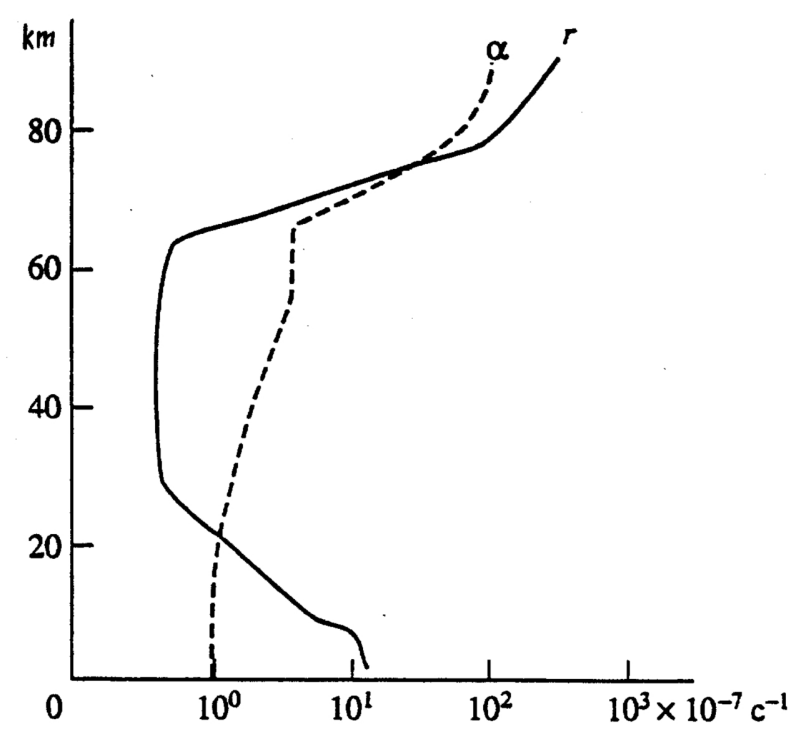

Fig. 7. Vertical structure of Newtonian cooling $(\alpha)$ and Rayley friction $(r)$ coefficients which were used for model calculations.

show the different sensivity of the middle atmosphere (at fixed spatial structure of the external forcing) to different periods of exitation. The peaks for 5-7, 13-14 and 24-26 days are the most steady and characterize the middle atmosphere as a whole. The amplitudes of these resonant waves increase with height reaching 60-90 gpm in the lower mesosphere. The phase of the most waves with resonant periods does not change strongly with height looking like free atmospheric modes usually look. The results of model calculations for different zonal wave numbers and frequencies has shown also that these waves penetrate to the lower latitudes at the higher levels in spite of the high latitudinal forcing. This effect is determinated by the reflection of the waves in a presence of zonal wind field with its real structure. The magnitudes for $m=2,3$ were smaller in comparison to the first harmonics.

The results of calculations for a second scenario has revealed the picture which was similar to previous one. Solar activity effects were switched out here and the middle atmosphere was exited from 


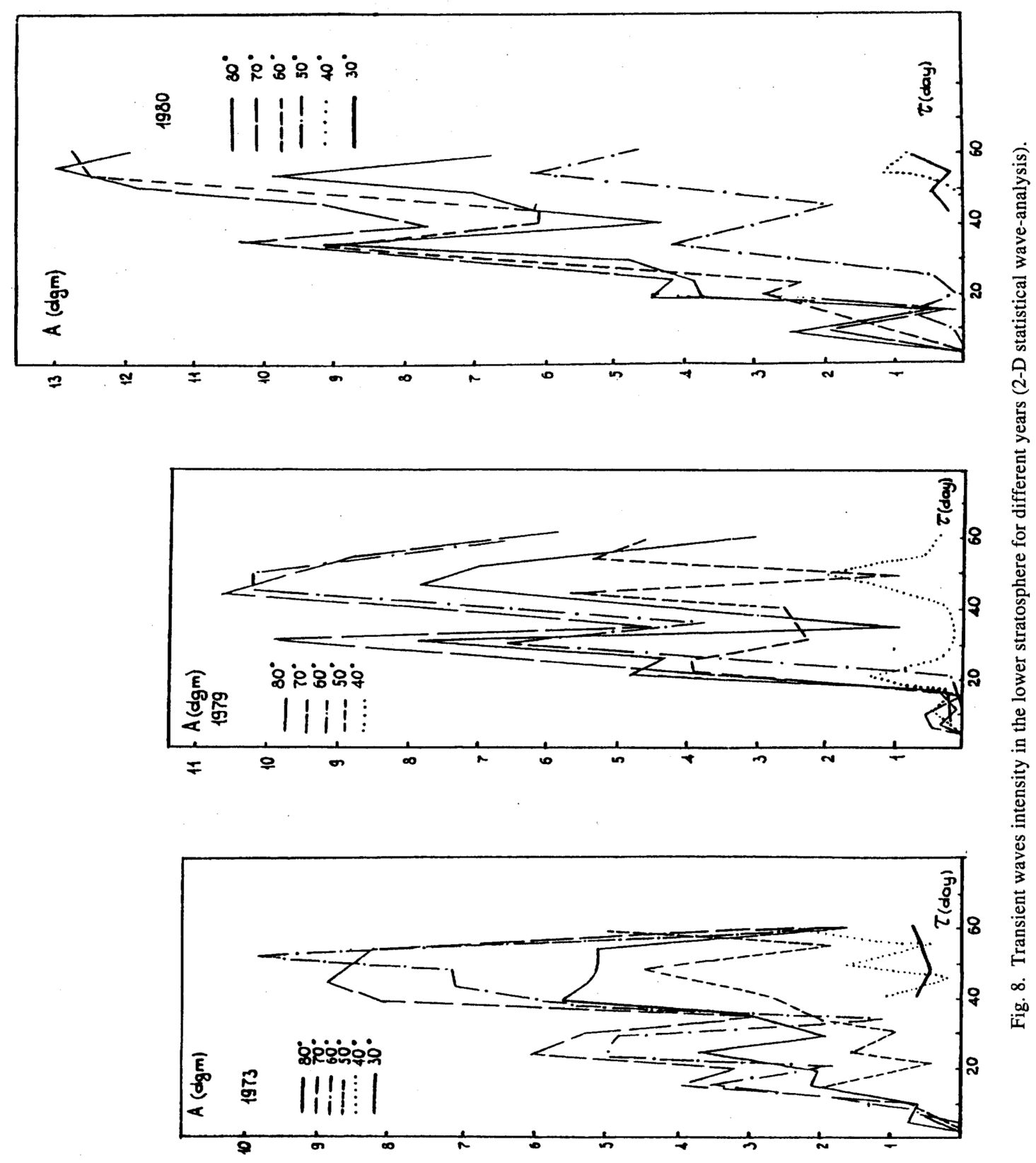


below due to the wave forcing at the lower boundary of integration ( $500 \mathrm{mb}$ level). The amplitude of such forcing was equaled $20 \mathrm{gpm}$ in accordance to data spectral analysis results. The structure of such exitation also looked like resonant one and with a good penetration of these waves to the higher levels. This effect is rather natural when the Earth's atmosphere has its own character periods as a whole. There is no principal difference between the displacement of excitement in such case but the amplitude of this excitement is important in the presence of real atmospheric viscosity.

Figure 8 shows the results of data analysis which revealed large-scale transient waves in the Northern Hemisphere. The daily gridded data of the geopotential heights for different years (without summer seasons) has been analised on base of a special statistical procedure (Krivolutsky, 1981). The results demonstrate the transient waves existance as a character feature of the middle atmosphere behaviour with the periods similar to the model calculations results.

\section{Conclusions}

The 2-D numerical study of the middle atmosphere sensitivity to the external periodical forcing has revealed the character periods of a higher response for 5-7, 12-14, 25-30 and 40-50 days. Large-scale transient waves with these periods may be generated possibly by tropospheric disturbances or solar ultraviolet periodical variations when the absorption has non-zonal climatological behaviour. So that the efficiency of solar-atmospheric link of this type depends not only on solar signal but also on behaviour of the middle atmosphere as a resonant system and its non-zonal features. The results of data analysis based on aerological information revealed similar transient planetary waves structure but it is actual to realize a complex study using UV variability, global ozone structure and middle atmosphere parameters based on satellites information jointly with numerical simulations

\section{REFERENCES}

Diky, L., The Earth's Oscillations Theory, 195 pp., Hidrometeoizdat, Leningrad, 1969.

Diky, L. and G. Golitsyn, Calculation of the Rossby wave velocities, Tellus, 20, No. 1, 156-163, 1968.

Ebel, A., B. Schwister, and K. Labitzke, Planetary waves and solar activity in stratosphere between 50 and 10 mb., J. Geophys. Res., 86, 9729-9738, 1981.

Ebel, A., M. Dameris, H. Hass, A. H. Manson, C. E. Meek, and K. Petzoldt, Vertical change of the response to solar activity oscillations with periods around 13 and 27 days in the middle atmosphere, Ann. Geophys., 4, A4, 271-280, 1986.

Eliassen, E. and B. Machenhauer, A study of the fluctuation of the atmospheric planetary flow patterns represented by spherical harmonics, Tellus, 17, No. 2, 220-238, 1965.

Garsia, R. and S. Solomon, A numerical model of the zonally averaged dynamical and chemical structure of the middle atmosphere, J. Geophys. Res., 78, 1379-1400, 1983.

Gille, M. C., C. M. Smythe, and D. F. Heath, Observed ozone response to variations in solar UV radiation, Science, 225, 315$317,1985$.

Haurwitz, B., The motion of atmospheric disturbances on the spherical earth, J. Marine Res., 3, 254-287, 1940.

Heath, D. F., A review of observational evidence for short and long-term UV flux variability of the sun, in Sun and Climate, pp. 447-471, CNES, 18 Ave. Edouard-Berlin, 31055-Tolouse, Cedex, France, 1980.

Hood, L. L., The behaviour of upper stratospheric ozone at low latitudes: evidence from NIMBUS4 BUV data for short term response to solar ultraviolet variability, J. Geophys. Res., 89, 9557-9568, 1984.

Hood, L. L., Solar ultraviolet radiation induced variations in the stratosphere and mesosphere, J. Geophys. Res., 92, D1, 876-885, 1987.

Ivanovsky, A. and A. Krivolutsky, About the possibility of a planetary waves resonant exitation, Meteorologia and Gidrologia, No. 6, 97-99, 1979.

Kato, S., Diurnal atmospheric oscillations, 1, eigenvalues and Hough functions, J. Geophys. Res., 71, 3201-3216, 1966.

Keating, G. M., G. P. Brasseur, J. Y. Nicholson, and A. De Rudder, Detection of the response of ozone in the middle atmosphere to short term ultraviolet variation, Geophys. Res. Lett., 12, 449-452, 1985.

Keating, G. M., M. Pitts, G. Brasseur, and A. De Rudder, Response of the middle atmosphere to short-term solar ultraviolet variations, J. Geophys. Res., 92, D1, 889, 1987.

Keckhut, P. and M. L. Chanin, Middle atmosphere response to the 27-day solar rotation as observed by lidar, Geophys. Res. Lett., 19, No. 8, 809-812, 1992. 
King, J. W., A. J. Slater, A. D. Stevens, P. A. Smith, and D. M. Willis, Large-amplitude standing planetary waves induced in the troposphere by sun, J. Atmos. Terr. Phys., 39, 1357-1367, 1977.

Krivolutsky, A. A., Data analysis for transient waves detection, Meteorologia and Gidrologia, No. 10, 102-104, 1981.

Krivolutsky, A. A., Atmospheric planetary waves induced by solar rotation, Handbook for MAP, 29, 86-91, 1989.

Krivolutsky, A. A. and B. M. Kiryushov, The 2-D numerical modelling of the dynamical input for the middle atmosphere due to 27-days UV variability, General Assembly of EGS, Edinburg, U.K., Report, 1992.

Krivolutsky, A. A. and O. A. Loshkova, Resonant Rossby waves and solar activity, Handbook for MAP, 29, 92-94, 1989.

Krivolutsky, A. A., V. N. Kuznetsova, and D. A. Tarasenko, Wave motions in the middle atmosphere in relation to the solar activity, Physika Scripta, 36, 382-384, 1987.

Leovy, C., Some energy sources and sinks in the upper atmosphere, in Atmospheres of Earth and Planets, edited by B. M. D. McCormac, pp. 73-86, Reidel Publ. Co., Dordrecht, The Netherland, 1975.

Lindzen, R., The application of classical atmospheric tidal theory, Proc. Roy. Soc., A303, 299-316, 1968.

Longuet-Higgins, M., The eigenfunctions of Laplace's tidal equations over a sphere, Phil. Trans. Roy. Soc., A262, 511-607, 1968.

Madala, R. V., An efficient direct solver for separable and non separable eliptic equations, Mon. Wea. Rev., 106, 1735-1741, 1978.

Shoeberl, M. and J. Clark, Resonant planetary waves in a spherical atmosphere, J. Atmos. Sci., 37, 20-37, 1980.

Shoeberl, M. and D. Strobel, The zonally averaged circulation of the middle atmosphere, J. Atmos. Sci., 35, 577-591, 1978.

Smirnov, R. V., The tendency to 27-day periodicity in temperature field of troposphere, Trudy MGI, 37, 163-169, 1966.

Vitels, L. A., The solar nature of atmospheric cycles, Trudy CIP, 51, 22-43, 1957.

Volland, H., Periodic variations of solar radiation-a possible source of solar activity-weather effects, J. Atmos. Terr. Phys., 41, 89-94, 1979. 